\title{
SISTEMATIZACIÓN DEL DESCUBRIMIENTO Y LA EXPLICACIÓN: LA ELABORACIÓN DE UNA LÓGICA ABDUCTIVA
}

Atocha Aliseda, Abductive Reasoning. Logical Investigations into Discovery and Explanation, Springer, Dordrecht, 2006 (Synthese Library, 330), xvi + 225 pp.

\author{
Ángel Nepomuceno Fernández \\ Departamento de Filosofía y Lógica \\ Universidad de Sevilla \\ nepomuce@us.es
}

\section{Introducción}

Hay una variedad de definiciones acerca de qué es la inferencia abductiva, forma de inferencia científica por excelencia: reverso de la deducción, inferencia de los efectos a las causas, razonamiento explicativo, etc. Esta diversidad de nociones reclama estudios sistemáticos que traten de fijar satisfactoriamente este concepto, presente en importantes áreas de investigación como la inteligencia artificial, la lingüística y la filosofía de la ciencia, entre otras. Ésta es la tarea que se pretende llevar a cabo en la obra Abductive Reasoning. Logical Investigations into Discovery and Explanation, de Atocha Aliseda. Este volumen es ya una referencia obligada para el estudio sistemático de la abducción desde varias ópticas: lógica, filosofía de la ciencia, computación, etc. Baste destacar, a este respecto, los comentarios laudatorios de Ilkka Niiniluoto en su ponencia magistral presentada en el Congreso de la Sociedad de Lógica, Metodología y Filosofía de la Ciencia celebrado en Granada (2006). Gabbay y Woods, en su A Practical Logic of Cognitive Systems (2006), identifican el "modelo AKM" como la propuesta por excelencia para el tratamiento de la abducción, y estas siglas corresponden a "Aliseda", "Kakas/Kowalski/Kuipers" y "Magnani/Meheus", los autores de estos enfoques. Asimismo, recientemente se ha publicado un número de la revista Theoria, donde se presenta un foro que consta de diez contribuciones críticas sobre el tema de este libro (Teoria, vol. 22-23, no. 60, septiembre de 2007). 
Si el punto de partida para establecer un modelo fue la primera obra de la autora sobre esta temática (Aliseda 1997), hacía falta completarlo con este volumen que, por otra parte, resulta claramente novedoso, pues aparecen nuevos conceptos, análisis, desarrollos, críticas y referencias.

En este trabajo me propongo estudiar algunos de los planteamientos abordados por la autora. A un consenso básico en cuestiones fundamentales, hay que añadir discrepancias que alimentan, por así decirlo, saludables disputas benéficas para el saber. La sociedad del conocimiento así lo reclama. A modo de ejemplo, la representación de la abducción como inferencia lógica es cuestionable, así como un tratamiento tan estrechamente unido a la lógica clásica, aun cuando su pretensión sea abarcar todas las lógicas. Tras esta introducción, presento un breve resumen de la obra. En la sección 3 me ocupo de cuestiones tratadas por la autora principalmente en los capítulos 3 y 4 del volumen, lo que se completa en la sección 4, donde, constatada la limitación a la lógica clásica como base para el estudio lógico de la abducción, propongo nuevos abordajes teniendo en cuenta lógicas modales y lógicas no clásicas. Finalmente se incluyen referencias bibliográficas.

\section{Resumen de la obra}

El libro se estructura en tres partes, con un total de ocho capítulos, prólogo, índice de autores e índice de materias, además de las referencias bibliográficas, con 223 entradas, ordenadas alfabéticamente por autores y etiquetadas con un código alfanumérico. El objetivo propuesto, un estudio de la abducción, se plantea a partir de presupuestos que se concretan en la inexistencia de un método lógico simple en la práctica científica, se piensa en ciencia normal, y hay que tener en cuenta lo artificioso de la distinción entre contextos de descubrimiento y de justificación.

En el capítulo 1, la autora considera que cualquier descubrimiento involucra un proceso que abarca desde la concepción inicial de una idea, justificaciones de la misma, hasta su establecimiento como una teoría. Se repasan diversas concepciones para poner de relieve la dificultad de delimitar los contextos, cuestionar si cabe la reflexión filosófica sobre los mismos y su estudio lógico. Atendiendo a antecedentes históricos, la lógica no permite la completa caracterización de nociones propias de la filosofía de la ciencia, lo que no excluye que algunos problemas en la historia de la ciencia se puedan abordar desde un punto de vista formal; de hecho, lo histórico y lo 
formal están en buena relación en la llamada filosofía computacional de la ciencia.

En el capítulo 2 se intenta responder a la pregunta: ¿qué es abducción? Será producto o proceso, construcción o selección, no una mera inducción, como una forma de razonamiento que va de una simple observación a sus explicaciones abductivas, distintas de la inducción enumerativa que parte de ejemplos para alcanzar afirmaciones generales. También aparece el planteamiento original de Peirce y su estructura lógica: una relación ternaria con una teoría, una observación y una explicación como argumentos de la misma. Se presentan tres parámetros abductivos: el inferencial, los desencadenantes o disparadores (de novedad abductiva y anomalía abductiva) y, por último, los diferentes tipos de resultados.

En el capítulo 3, Aliseda trata de caracterizar lógicamente los argumentos explicativos (abductivos), para lo cual adopta el método de análisis estructural y presenta varios estilos de abducción: plana, consistente, explicativa, minimal y preferencial. ${ }^{1}$ A partir de un lenguaje proposicional se constata cómo fallan las reglas de reflexividad, monotonía y corte, si bien se verifican otras.

El estudio de la abducción como computación se aborda en el capítulo 4, retomando la idea de que se trata de un proceso, y se destacan dos marcos vigentes: los de la programación y las tablas semánticas. El objetivo ahora es mostrar cómo se implementa la abducción, siguiendo una de las siguientes vías: a) análisis como los realizados en teoría de la demostración o en sistemas lógicos especiales, b) la programación de algoritmos puramente computacionales. Se presenta asimismo un estudio del método de tablas semánticas, tanto las estándar como algunas variantes y su aplicación en la búsqueda de soluciones a problemas abductivos.

La tercera y última parte está dedicada a las aplicaciones, divididas en cuatro capítulos. El capítulo 5 se ocupa de la explicación científica y estudia las propuestas de Hempel y Oppenheim, tanto el modelo nomológico-deductivo como el estadístico-inductivo. Con el nombre genérico de progreso empírico, en el capítulo 6 se proponen aplicaciones de lo estudiado en el capítulo 4 y se dirige la atención a los estudios de esta noción en Kuipers, aunque también se tienen en cuenta los puntos de vista de Laudan y Gärdenfors, y se aplica, a todas estas cuestiones, la metodología de tablas semánticas adaptadas para la abducción.

\footnotetext{
${ }^{1}$ Más adelante nos ocupamos con mayor detenimiento de algunos contenidos de los capítulos 3 y 4 .
} 
El objeto del capítulo 7 es realizar un breve estudio del pragmatismo, en la formulación que de éste hace Peirce, como método de reflexión que aspira a esclarecer ideas; tal método propone un objetivo epistémico siguiendo la máxima pragmática (fijar una creencia y producir sus consiguientes hábitos de acción). Para concluir, en el capítulo 8, último del libro, la autora se ocupa de estudiar el cambio epistémico a partir del modelo clásico AGM (Alchourrón, Gärdenfors y Makinson). Ahí se muestra cómo el razonamiento abductivo brinda un modelo de cambio epistémico, de manera que los dos disparadores abductivos se ponen en correspondencia con las actitudes epistémicas de indeterminación o rechazo de una fórmula.

\section{Abducción, lógica y computación}

Una importante cuestión para abordar la abducción desde un punto de vista lógico es fijar qué se entiende por sistema lógico, punto ciertamente importante estudiado por la autora en el capítulo 3. A la base estaría la atractiva clasificación de Peirce de los tipos de inferencia: deductiva, inductiva y abductiva. Sin embargo, no se trata a fondo dónde encajar inferencias de suma importancia en la investigación científica; por ejemplo, los modos de obtener conclusiones cuando se trabaja en minería de datos, o el razonamiento estadístico en general.

Al formular una hipótesis explicativa, lo que se exige es que ésta sea plausible. ¿Es posible entonces hablar de sistemas lógicos que den cuenta ello? A su estudio presta Aliseda la máxima atención. Los sistemas lógicos habituales captan perfectamente la inferencia deductiva y es pertinente la adopción del método de análisis estructural; éste, como señala la autora, se basa en la sencilla idea según la cual una noción de inferencia lógica puede ser caracterizada completamente por sus propiedades combinatorias básicas, expresadas mediante reglas estructurales. Este procedimiento, en el que se conjugan la máxima precisión y una inusitada sencillez, permite definir la relación de consecuencia lógica clásica mediante sus reglas estructurales características: reflexividad, contracción, permutación, monotonía y corte. Éste es el camino seguido por Aliseda, justificado en opiniones de una pléyade de autores de reconocida autoridad. ${ }^{2}$

En la inferencia deductiva, estamos claramente ante una relación diádica, con un conjunto de fórmulas como input (entrada) y una

${ }^{2}$ Para fijar la noción de sistema formal, se tiene en cuenta el punto de vista de Haack, así como los de Kneale, Dummett y Hintikka. La justificación del análisis estructural se apoya en autores como Scott, Tarski, Schroeder-Heister y Dosen, Kraus, Lemman y Magidor, Gabbay y van Benthem. 
fórmula como output (salida). Aliseda opta por considerar la inferencia abductiva como una relación con carácter ternario, y la representa mediante el símbolo " $\Rightarrow$ ". Dadas una teoría (un conjunto de fórmulas de un lenguaje) $\Theta$ y un hecho (una fórmula) $\varphi$, que constituyen un problema abductivo, se trata de hallar una fórmula $\alpha$, que sea una solución a dicho problema, una explicación de $\varphi$ (con la teoría $\Theta$ ). La definición (p. 75) es la siguiente: " $\Theta \mid \alpha \Rightarrow \varphi$ si y sólo si (i) $\Theta, \alpha \models \varphi$ y (ii) $\Theta, \alpha$ son consistentes", y desde aquí se propone una reducción a relación diádica agrupando $\Theta$ y $\alpha$ en un único conjunto como primer argumento, y $\varphi$ como segundo. Respecto de la abducción consistente, sus reglas estructurales serán reflexividad condicional, corte simultáneo y consistencia de la conclusión, y se establece un teorema de representación y algunas reglas conocidas, aunque mínimamente modificadas.

Cabe presentar una pequeña objeción a esta manera de visualizar la inferencia abductiva. Los datos de entrada y salida deberían aparecer a izquierda y derecha de $\Rightarrow$, respectivamente; es decir $(\Theta, \varphi) \Rightarrow \alpha$, que respeta el orden "natural" de la abducción. Es verdad que una definición como " $(\Theta, \varphi) \Rightarrow \alpha$ si y sólo si (i) $\Theta, \alpha \models \varphi$ y (ii) $\Theta$, $\alpha$ son consistentes" hace equivalentes ambas representaciones. En cualquier caso, la abducción aparece estrechamente ligada a la deducción. Se proponen varios estilos, según qué se verifique:

1. Plana

(i) $\Theta, \alpha \models \varphi$

2. Consistente

(i) $\Theta, \alpha \models \varphi$

(ii) $\Theta, \alpha$ son consistentes

3. Explicativa

(i) $\Theta, \alpha \models \varphi$

(ii) $\Theta \not \varphi$

(iii) $\alpha \neq \varphi$

4. Minimal

(i) $\Theta, \alpha \models \varphi$

(ii) $\alpha$ es la explicación abductiva más débil (no igual a $\Theta \rightarrow \varphi$ ) 
5. Preferencial

(i) $\Theta, \alpha \models \varphi$

(ii) $\alpha$ es la mejor explicación abductiva de acuerdo con algún orden preferencial dado

Destaca la tercera, que unida a la segunda constituye la "abducción explicativa consistente":

(i) $\Theta, \alpha \models \varphi$

(ii) $\Theta, \alpha$ son consistentes

(iii) $\Theta \nRightarrow \varphi$

(iv) $\alpha \not \varphi$

Cuestiones importantes son las de la completud, la complejidad y el papel del lenguaje. Sobre la primera, advierte la autora que los argumentos de representación para caracterizar la abducción fácilmente pueden ser reformulados en teoremas de completud si estamos ante un lenguaje sin operadores lógicos; cuando se toma otro más rico (añadiendo estos operadores), los argumentos de completud necesitan una mejora de las representaciones usadas, y en algún nivel no cabe ya esperar más resultados de completud, atendiendo a la complejidad de las pruebas. Se da una razón a favor del uso de un lenguaje proposicional: se expresa así una abducción todavía decidible y un alegato a favor de considerar como lógica deductiva básica la lógica clásica, dado que, de otro modo, hallaríamos un incremento de complejidad que también afectaría a la correspondiente lógica abductiva. Sin embargo, un lenguaje proposicional es expresivamente pobre y hay fragmentos de lenguajes de predicados de primer orden (y de segundo) que permiten definir lógicas decidibles. Al carácter básico otorgado a la lógica clásica volveremos en el próximo apartado.

A partir de la proposición "todas las nociones de inferencia explicativa abductiva estudiadas son decidibles sobre cláusulas universales" (p. 87), sustentada en el análisis estructural, se concluirá que cabe obtener representaciones de la abducción como sistemas lógicos. En última instancia, todo depende de cómo se responde a la pregunta: ¿qué es la lógica? Aliseda ofrece argumentos a favor de una lógica abductiva, recurre a la analogía de las geometrías no euclídeas. Su comparación con el cálculo inductivo de Flach (1995) muestra algunas similitudes y diferencias; pero si cabe hablar de lógica inductiva, 
una lógica subestructural (no por ello de carácter "deductivo"), como lo son los sistemas abductivos representados en esta obra, ¿por qué no considerarlos entonces sistemas de lógica abductiva? Atendiendo a importantes propiedades metateóricas (decidibilidad, corrección, completud) o de filosofía de la lógica, un punto de vista opuesto defendería la lógica de predicados de primer orden con identidad como la única lógica; en esta misma línea estará quien defienda como exclusiva lógica genuina, por ejemplo, la lógica intuicionista; pero se trata de una perspectiva monista. Aun se puede añadir algún ejemplo más a favor de la posición pluralista. Los sistemas de lógica proposicional son decidibles, correctos y completos; los sistemas de lógica de predicados de primer orden son correctos y completos, pero no decidibles; los sistemas de cálculo de predicados de segundo orden (o superior) son correctos, pero no completos (en sentido estándar) ¿No estamos en los tres casos en el ámbito de la lógica? En definitiva, cada relación de consecuencia estructurada es representable como un sistema lógico. ${ }^{3}$

El tratamiento de la abducción como computación, aparte de constatar que la programación lógica constituye un marco para la computación de la abducción como proceso, es una apuesta de la autora en la que, a pesar de la semidecidibilidad de la lógica clásica de primer orden, va más allá de los lenguajes proposicionales. Para el uso de tablas semánticas, el planteamiento es muy sencillo: sea el problema abductivo $(\Theta, \varphi)$, donde $\Theta$ es un conjunto de cardinalidad finita; a partir de $\Theta \cup\{\neg \varphi\}$ se construye la tabla semántica, si ésta es abierta (precondición de ser realmente un problema abductivo), entonces se puede construir una fórmula $\alpha$ tal que $\Theta \cup\{\alpha, \neg \varphi\}$ tiene una tabla cerrada, es decir, $\Theta, \alpha \models \varphi$. A nivel proposicional, no hay problema alguno, pero si el lenguaje es de primer orden, entonces nos topamos con la indecidibilidad, $\Theta \cup\{\neg \varphi\}$ puede dar lugar a ramas infinitas, lo cual no es óbice para estudiar casos especiales, cuando la precondición no plantea problemas de indecidibilidad. Hay que aplaudir este planteamiento, lo que supone un avance, al margen de otras diferencias, respecto de la obra anterior de la autora (Aliseda 1997). Si se me permite la analogía, así como la certeza en la finitud del hombre no ha impedido el desarrollo de la medicina geriátrica, la certeza de que se pueden presentar problemas abductivos con tal

${ }^{3}$ En Nepomuceno y Soler 2007 se representa la relación de consecuencia explicativa (abductiva) entre conjuntos de fórmulas $A$ y $B: A \models \alpha B, A$ es explicado por $B$, lo cual equivale a que, para toda $a \in A$, se verifica $B \models a$ ( $a$ es consecuencia lógica de $B$ ). 
precondición como indecidible no tiene por qué impedir el estudio de casos especiales de precondiciones tratables con tablas semánticas modificadas; aunque, siguiendo con la analogía, si no se trata de extender la vida a cualquier precio, en lógica debemos conocer las limitaciones de un sistema, saber, por ejemplo, cuándo no es posible exigir completud.

El uso de tableaux, en definitiva, se puede adoptar también en el nivel de primer orden para la búsqueda de soluciones a problemas abductivos. Dada una precondición $\Theta \cup\{\neg \varphi\}$, la fórmula resultante de la conjunción de las que integran dicha precondición podría pertenecer a una clase con la propiedad de modelos finitos. Precisamente la modificación de la regla $\delta$ de las tablas estándar (de Beth o de Smullyan) permite obtener modelos mínimos de fórmulas de algunas de estas clases. Se les podría sacar más partido a estos procedimientos, por así decirlo, en la medida en que las tablas modificadas permitieran abordar problemas del ámbito de la teoría de modelos finitos. Baste mencionar como ejemplo el caso de fórmulas prenexas que tienen la forma $\forall^{2} \exists^{*} \varphi$, cuyo prefijo consta de dos cuantificadores universales seguidos de un número indeterminado (naturalmente finito) de cuantificadores existenciales, y $\varphi$ representa la fórmula matriz ( $\sin$ cuantificadores); asimismo, se pueden estudiar casos de lógica monádica de segundo orden, ${ }^{4}$ etc. Quedaría pendiente un estudio de las clases de complejidad en relación con la abducción, así como la posibilidad de definición de nuevos cálculos lógicos abductivos. Me ocuparé brevemente de lo segundo en la siguiente sección.

En cuanto a la limitación, en el nivel cuantificacional, de obtener sólo literales como abducciones, Aliseda la señala y sostiene que es posible extender el procedimiento para obtener fórmulas más complejas. De hecho, en el nivel proposicional se planteaban abducciones conjuntivas y disyuntivas. Un desarrollo en este sentido podría modelar ciertos procesos abductivos. Para simplificar veamos un ejemplo muy sencillo. Sea una teoría que consta de $\forall x(P x \rightarrow Q x)$ como única fórmula; consideremos el hecho representado por $P a$, y supongamos que se nos presenta el hecho "sorprendente" $S a$. Si buscamos una explicación mediante tableaux, a partir de la raíz formada por $\forall x(P x \rightarrow Q x)$, junto con $P a$ y $\neg S a$, obtenemos una rama abierta que contiene los literales $P a, \neg S a$ y $Q a$, por lo que el conjunto de abducibles (literales que proporcionan cierres de la rama abierta) será $\{\neg P a, S a, \neg Q a\}$; desechamos $\neg P a$ (dado que $P a$ viene a ser

\footnotetext{
${ }^{4}$ En Ebbinghaus y Flum 1991 se encuentra un desarrollo de la teoría de modelos finitos.
} 
una condición inicial), con lo que dicho conjunto queda reducido y la disyunción de todos sus literales permite obtener una tabla cerrada, por lo que podríamos tomar $\neg Q a \vee S a$ como solución y esta fórmula equivale a $Q a \rightarrow S a$ ¿Por qué no considerar una generalización a partir de ésta, es decir, $\forall x(Q x \rightarrow S x)$, como solución al problema abductivo planteado? O bien, ¿no sería una buena solución estipular la equivalencia de los predicados $S$ y $Q$, esto es, la fórmula $\forall x(Q x \leftrightarrow S x)$ ? Naturalmente ello requiere un mayor desarrollo de las formas de aplicación de tableaux en abducción, especialmente cuando se trata de seleccionar las soluciones más razonables de entre las alternativas que se presentan.

\section{Más allá de la lógica clásica}

Las tesis definitorias de la abducción dadas en Hintikka (1999) no fijan explícitamente la necesidad previa de una noción de deducción. No obstante, se insiste en que la abducción es, o incluye, un proceso (o varios) de carácter inferencial (tesis inferencial), aunque globalmente considerada es irreductible a deducción o inducción (tesis de autonomía). La taxonomía de estilos presentada, salvo en el caso de la abducción plana, muestra modos lógicos de entender la abducción que satisfacen las condiciones incluidas en dichas tesis. Como hemos observado, en todas las clases de abducción vistas en tal taxonomía subyace una noción de inferencia deductiva ¿Por qué adoptar exclusivamente la noción de deducción específica de la lógica clásica? ¿No sería preferible partir de una relación de consecuencia deductiva más general, o considerar varias relaciones a un tiempo, o, en último término, minimizar la exigencia de la tesis inferencial?

La relación de consecuencia lógica clásica, como se ha indicado líneas atrás, se caracteriza por ciertas reglas estructurales (reflexividad, monotonía, permutación, contracción y transitividad o corte) y, además, verifica sustitución uniforme y compacidad. Naturalmente resulta cómodo tomar una noción de deducción clásica como telón de fondo para el estudio lógico de la abducción, sobre todo si los cálculos que dan cuenta de aquélla son utilizables para "calcular" soluciones a problemas abductivos. Es cierto que las aplicaciones que se han hecho de los cálculos conocidos para la obtención de soluciones a problemas abductivos parecen exigir determinadas características a los cálculos en cuestión. Éstas se pueden resumir de la siguiente manera. Estando en el estilo clásico de inferencia, se considera una relación de teoremicidad (de deducibilidad) $\vdash$ de clausura la cual es 
correcta y se establecen condiciones definitorias de problema abductivo $(\Theta, \varphi)$, como, por ejemplo, que no sea el caso que $\Theta \vdash \varphi$ y tampoco sea el caso que $\Theta \vdash \neg \varphi$; asimismo, cabe considerar una relación ternaria: $\Re(\Theta, \alpha, \varphi)$ si y sólo si se cumplen tres requisitos: 1) $\Theta$ y $\alpha$ son consistentes, 2) no es el caso que $\alpha \vdash \varphi$, 3) la negación y la implicación están definidas de tal modo que en $\vdash$ se incluye contraposición y se cumple el (meta)teorema de la deducción. Entonces un cálculo abductivo es aquel que cumple la siguiente regla de inferencia abductiva: si $(\Theta, \varphi)$ es un problema abductivo (entrada) y $\Re(\Theta, \alpha, \varphi)$, entonces $\alpha$ es una solución (salida). ¿No se establece como condición indispensable que $\vdash$ sea completa? En realidad no es necesario, basta que sea correcta y, en todo caso, que se cuente con modus ponens, se verifique el teorema de la deducción y se tenga contraposición (o bien "contradicción implica trivialidad" y la consequentia mirabilis) (Nepomuceno y Soler 2009).

Si una relación de consecuencia cumple reflexividad, monotonía y transitividad, decimos que es una relación de clausura. La relación de consecuencia lógica clásica es una relación de clausura, pero cabe pensar en relaciones de clausura distintas de la prototípica relación clásica, cuando, por ejemplo, no verifican compacidad o sustitución uniforme. En general, las relaciones de clausura se mantienen en lo que podríamos llamar el estilo clásico, es decir, se trata de relaciones de consecuencia $\models$ que serán de clausura, aunque no cumplan sustitución uniforme ni compacidad. Como hemos visto, basta hallar cálculos (relaciones de teoremicidad $\vdash$ ) que sean correctos, aun cuando no sean completos (no podrían serlo si $\models$ no verifica compacidad). De este modo, podemos trabajar con relaciones de consecuencia lógica que, en sentido estricto, no son propiamente la clásica, en la línea seguida en Makinson 2005, a saber, relaciones de consecuencia módulo un conjunto de fórmulas y módulo un conjunto de modelos. También son definibles relaciones de consecuencia módulo un conjunto de reglas adicionales, que permiten modelar lógicas no monótonas normales, por ejemplo. Brevemente, sean $\mathrm{F}$ un conjunto de fórmulas y M un conjunto de modelos, entonces

1) $\varphi$ es consecuencia lógica módulo $\mathrm{F}$ de $\Theta$, simbólicamente $\Theta \models$ (F) $\varphi$, si y sólo si se verifica que $\Theta \cup F \models \varphi$

2) $\varphi$ es consecuencia lógica módulo $\mathrm{M}$ de $\Theta$, simbólicamente $\Theta \models(\mathrm{M}) \varphi$, si y sólo si se verifica que todos los modelos de M que satisfacen $\Theta$, también satisfacen $\varphi$ 
Una variante se obtiene si M representa la clase de los modelos cuyo universo de discurso es finito. Otra, cuando $\mathrm{M}$ es la clase de modelos cuyo universo de discurso tiene un número (finito) determinado de elementos. En todas las variantes falla la sustitución uniforme y, en algunos casos, la compacidad. Se trata, en suma, de diferentes relaciones de consecuencia lógica que se determinan apelando a clases de fórmulas o a clases de modelos, es decir, a los contextos (inferenciales) que tales clases representan — una suerte de consecuencia "sensible al contexto"-. Ahora bien, son definibles cálculos (deductivos) correctos, no siempre completos, pero permiten definir cálculos abductivos también correctos. Lo mismo cabe decir si nos situamos en el nivel de segundo orden (o superior) con semántica estándar.

Si pensamos en la inferencia propia de las prácticas científicas, también cabe plantear lo que podríamos denominar problemas abductivos estructurales: ${ }^{5}(\Theta, \varphi)$ constituye un problema abductivo estructural cuando se trata no tanto de buscar una fórmula adicional $\alpha$, como de hallar la lógica más adecuada, la correspondiente relación de teoremicidad $\vdash$, tal que $\Theta \vdash \varphi$, bien porque la lógica inicial no cuenta con reglas suficientes que justifiquen la inferencia ya asumida en la práctica científica - podría haber razones específicas del campo de que se trate que no están captadas en las lógicas habituales-, bien porque se descubre la necesidad de modificar alguna regla, etc. Tal vez en el caso de las lógicas modales se muestra todo ello con la mayor nitidez. Sea $\vdash$ la relación que define un sistema modal normal; para cada mundo $w$, un conjunto de fórmulas $\Theta$ y una fórmula $\varphi$, se verifica que $\Theta, w \vdash \varphi$, lo que indica que $\varphi$ es demostrable desde $\Theta$ en el mundo $w$, o no es el caso. Entonces, dados $\Theta$ y $\varphi$, diremos que constituyen un problema abductivo estructural en un mundo $w$ cuando $\varphi$ es independiente de $\Theta$ en dicho mundo, es decir, no se da $\Theta, w \vdash \varphi$ ni $\Theta, w \vdash \neg \varphi$, y se trata de hallar un mundo $u$ (si ello es factible) tal que $\Theta, u \vdash \varphi$. Precisamente este tipo de precondición lleva a una formulación modal del problema mismo que puede resultar interesante desde un punto de vista epistemológico: el par $(\Theta$, $\varphi)$ se considera problema abductivo en un mundo $w$ si (y sólo si) $\Theta, w \vdash \diamond \varphi$, al tiempo que $\Theta, w \vdash \diamond \neg \varphi$. Es decir, como abducción científica, para la teoría $\Theta$ y el hecho que se pretende explicar $\varphi$, se trata de que exista un contexto (un mundo) $u$, accesible desde el

${ }^{5}$ Tomamos esta noción siguiendo en parte el planteamiento dado en Keiff 2007, pp. 199 y ss. En cierto modo, la abducción estructural también aparece en D'Agostino et al. 2008, donde se contempla el caso de problema abductivo $(\Gamma, G)$ tal que se verifica $\Gamma \vdash \mathrm{G}$ y de lo que se trata es de hallar su demostración. 
dado, en el que $\varphi$ sea demostrable a partir de $\Theta$ en otro $u^{\prime}$ en el que $\neg \varphi$ sea demostrable a partir de $\Theta$ y la exigencia de consistencia de que $u$ y $u^{\prime}$ sean distintos. ${ }^{6}$

Otra versión consiste en examinar si en el mundo $w$ no se verifica que $\Theta, w \vdash \varphi$, con las características dadas a la relación de accesibilidad, entonces hallar una nueva relación (naturalmente con distintas características) tal que se alcance que $\Theta, w \vdash \varphi$. Un ejemplo sencillo consiste en considerar $\Theta=\{\diamond \beta\}$, la fórmula (esquema) $\square \diamond \beta$ y la relación de accesibilidad como reflexiva y simétrica; entonces $(\{\diamond \beta\}$, $\square \diamond \beta$ ) es un problema abductivo en cada mundo $w$, puesto que no se da que $\{\diamond \beta\}, w \vdash \square \diamond \beta$ ¿Cabe buscar una solución a este problema abductivo haciendo uso de alguno de los cálculos conocidos?

Aliseda no plantea este tipo de problemas; no obstante, para abordarlos, no hay más que llevar los métodos adoptados hasta sus últimas consecuencias. Las tablas semánticas eran la herramienta adecuada para una búsqueda sistemática de soluciones a problemas abductivos. Para lógicas modales hay que hacer ligeras modificaciones en las tablas; éstas se habrán de establecer de manera que, en cada nodo del grafo correspondiente, en lugar de una fórmula en solitario, aparezca la fórmula junto a un índice o mundo y, en algunos casos, aparecerán sólo expresiones de que ciertos mundos están relacionados, es decir, que pertenecen a la correspondiente relación de accesibilidad. Además se definen reglas relativas al carácter de esta relación, que anotamos como R. Todas las reglas de las tablas estándar se mantienen, teniendo en cuenta la notación indicada, y se establecen algunas reglas nuevas:

1) Reglas generales para lógicas modales normales:

Desde $\neg \square \beta$, $i$, continuar la rama con $\diamond \neg \beta, i$

Desde $\neg \diamond \beta$, $i$, continuar la rama con $\square \neg \beta, i$

Desde $\square \beta, i$ y $\mathbf{R} i j$, continuar la rama $\operatorname{con} \beta, j$

Desde $\diamond \beta, i$, continuar la rama con $\mathbf{R} i j$ y $\beta, j$

2) Para $\mathbf{R}$ reflexiva:

Anotar, para cada índice (o mundo) $i$ de la rama, $\mathbf{R} i$.

${ }^{6}$ F. Soler, en las II Jornadas Ibéricas de Lógica y Filosofía de la Ciencia (Lisboa, 2007), propuso un marco modal para modelar la abducción científica, con cuatro operadores modales en el lenguaje que contiene al lenguaje de las teorías. Así, los problemas abductivos se plantean como fórmulas (esquemas) concretas. 
3) Para $\mathbf{R}$ simétrica:

Desde $\mathbf{R} i j$, continuar la rama con $\mathbf{R} j i$.

4) Para $\mathbf{R}$ transitiva:

Desde $\mathbf{R} i j$ y $\mathbf{R} j k$, continuar la rama con $\mathbf{R} i k$.

En relación con el ejemplo señalado, considerando que se cumple la precondición, iniciamos la búsqueda de solución a este problema abductivo estructural; para ello construiremos una tabla a partir del esquema $\diamond \beta \wedge \neg \square \diamond \beta$, equivalente a $\neg(\diamond \beta \rightarrow \square \diamond \beta)$, resultante de negar el obtenido al aplicar el teorema de la deducción, en un mundo, que representaremos como 1 , asimismo se considera que $\mathbf{R}$ es reflexiva y simétrica. La tabla tiene una única rama, que puede presentarse así:

1) $\diamond \beta \wedge \neg \square \diamond \beta, 1$

2) $\mathbf{R} 11$

3) $\diamond \beta, 1$

4) $\neg \square \diamond \beta, 1$

5) $\mathbf{R} 12$

6) $\beta, 2$

7) $\diamond \neg \diamond \beta, 1$

8) $\mathbf{R} 13$

9) $\neg \diamond \beta, 3$

10) $\mathbf{R} 22$

11) $\mathbf{R} 33$

12) $\mathbf{R} 21$

13) $\mathbf{R} 31$

14) $\square \neg \beta, 3$

15) $\neg \beta, 1$

16) $\neg \beta, 3$ 
Aparecen $\mathbf{R} 11, \mathbf{R} 22$ y $\mathbf{R} 33$, pues $\mathbf{R}$ es reflexiva. Aquí termina la única rama (la única conectiva proposicional era $\wedge$ ), la cual resulta abierta ¿Cómo se obtiene un cierre de esta rama? Bastaría que apareciera $\mathbf{R} 32$, en cuyo caso se alcanzaría $\neg \beta, 2$, lo que entra en contradicción con lo expresado en la sexta línea. Si la relación de accesibilidad es transitiva, entonces, dado que aparecen $\mathbf{R} 31$ y R 12 , habría que anotar, según la regla correspondiente, R32, lo que lleva directamente a $\neg \beta, 2$. Así, en el ámbito de la lógica modal, la solución al problema abductivo estructural $(\{\diamond \beta\}, \square \diamond \beta)$, que se plantea en cada mundo cuando $\vdash$ representa una lógica normal cuya relación de accesibilidad es reflexiva y simétrica, tiene como solución (dado que 1 representa un mundo cualquiera, para cada mundo) el que la relación de accesibilidad sea también transitiva, es decir, hay que pasar a un sistema S5. De manera análoga, se puede plantear $(\{\diamond \beta\}, \square \diamond \beta)$ como problema abductivo estructural en cualquier mundo en $\mathrm{S} 4$; en este caso nos hallaremos ante una relación de accesibilidad reflexiva y transitiva y la tabla discurriría de la siguiente manera:

1) $\diamond \beta \wedge \neg \square \diamond \beta, 1$

2) $\mathbf{R} 11$

3) $\diamond \beta, 1$

4) $\neg \square \diamond \beta, 1$

5) $\mathbf{R} 12$

6) $\beta, 2$

7) $\diamond \neg \diamond \beta, 1$

8) $\mathbf{R} 13$

9) $\neg \diamond \beta, 3$

10) $\square \neg \beta, 3$

11) $\mathbf{R} 22$

12) $\mathbf{R} 33$

En este caso, si la relación $\mathbf{R}$ fuese simétrica, entonces, de acuerdo con las reglas, se podrían añadir $\mathbf{R} 21$ y $\mathbf{R} 31$, con lo cual, dada su transitividad desde el inicio, se añadiría R32, lo que permite añadir 
$\neg \beta, 2$, en contradicción con lo expresado en la línea sexta. Así, la solución se halla cambiando de $\mathrm{S} 4$ a S5.

De la misma manera, mutatis mutandis, se pueden estudiar problemas en otras lógicas; por ejemplo, en lógica epistémica, lógica deóntica, etc., es decir, en muchas lógicas modales o multimodales. De modo que si nos hallamos en una lógica epistémica básica $\vdash$, cuyo axioma propiamente epistémico expresa que $\left(K_{a} \varphi \wedge K_{a}(\varphi \rightarrow \psi)\right) \rightarrow$ $K_{a} \psi$ (si $a$ conoce $\varphi$ y $a$ conoce (si $\varphi \rightarrow \psi$ ), entonces $a$ conoce $\psi$ ), y se plantea el problema abductivo $\left(K_{a} \varphi, K_{a} K_{a} \varphi\right)$, la solución se hallaría pasando a una lógica epistémica en la cual sea válida la introspección positiva, es decir, asumiendo el esquema $K_{a} \varphi \rightarrow K_{a} K_{a} \varphi$; por lo tanto, pasando a una lógica ${ }^{7}$ de las conocidas como K45, KD45, S4 o S5. Otra cuestión es que nos encontremos ante un sistema multiagentes y se plantee el problema $\left(\Theta, K_{x} \beta\right)$, cuando $\vdash$ es un sistema de lógica epistémica y se trate de hallar si existe un agente $a$ tal que $\Theta$ $\vdash K_{a} \beta$. En cualquier caso, se pueden plantear también los problemas abductivos normales, como problemas de explicación, en el ámbito de estas lógicas.

Finalmente, en las prácticas científicas la lógica subyacente no siempre es la clásica o alguna de sus extensiones, sino que podría ser una lógica no clásica. La idea de que las "lógicas del descubrimiento" deben encontrar su expresión en los modernos sistemas de lógica concebidos en el ámbito de la Inteligencia Artificial (expresada por la autora en la p. 25) constituye una invitación al estudio de la aplicación de otras tablas semánticas para lógicas no clásicas, además de las indicadas para lógicas modales, y la modelación mediante las mismas de algunos procesos abductivos, aunque la caracterización de cálculos abductivos correctos no se ajuste plenamente a lo indicado antes para relaciones de clausura. ${ }^{8}$ Con objeto de simplificar, veamos brevemente dos casos representativos. Si bien en la semántica de la lógica de la relevancia nos topamos con problemas que no han sido plenamente resueltos (Palau 2002, p. 120), podemos presentar una sencilla adaptación de las reglas de formación de las tablas para lógica de la relevancia, siguiendo en parte a D'Agostino et al. 1999. Para ello, se definen las conectivas denominadas fusión y fisión, o y + , respectivamente, de manera que se dan las equivalencias

\footnotetext{
${ }^{7}$ Un compendio de sistemas de lógica epistémica se halla en Gochet y Gribomont 2006.

${ }^{8}$ En estas lógicas no es válido el esquema $A \rightarrow(\neg A \rightarrow B)$, falla monotonía, puesto que, en general, vale $A \vdash A$, pero si $B$ es distinta de $A$, no se verifica que $A, B \vdash A$; no obstante, consequentia mirabilis es aceptable y se verifica el teorema de la deducción.
} 
$A \circ B \equiv \neg(A \rightarrow \neg B)$ y $A+B \equiv \neg A \rightarrow B$. Asimismo, se tiene en cuenta el requisito de uso, de acuerdo con el cual se exige utilizar todas las premisas o supuestos en una demostración, es decir, en la obtención de los cierres de ramas. La fusión y la fisión son tratadas de manera semejante a la conjunción y la disyunción clásicas, mientras que la regla para conjunción (y negación de disyunción) no obliga a escribir consecutivamente las fórmulas componentes en el desarrollo de la rama de la tabla de que se trate, sino sólo una, con lo que se cumple el requisito de uso; en caso de necesitar el otro componente, ésta será una segunda aplicación de la regla, es decir, se tratará de un "segundo uso". De este modo, fórmulas cuya validez es rechazada desde el punto de vista de la lógica de la relevancia, al negarlas dan lugar a tablas cerradas clásicamente pero abiertas si se construyen según las modificaciones indicadas. Tal es el caso de los esquemas $A \rightarrow(B \rightarrow A), A \wedge \neg A \rightarrow B$ y $A \rightarrow B \vee \neg B$, cuyas negaciones equivalen a las traducciones $A \circ(B \circ \neg A),(A \circ \neg A) \circ \neg B$ y $A \circ \neg(B \vee \neg B)$, respectivamente; las tablas correspondientes, aunque cerradas clásicamente, no lo son de manera relevante. Sin embargo, la siguiente tabla es cerrada tanto clásicamente como de manera relevante $(x$ indica que la fórmula correspondiente ha sido usada en el curso de la demostración, es decir, de la construcción de la tabla).

1) $(\neg A+B) \circ(\neg B \circ A) \times$

2) $\neg A+B \times$

3) $\neg B \circ A \times$

4) $\neg B \times$

5) $A \times$

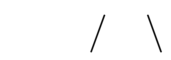

6) $\neg A \times \quad B \times$

$\otimes \otimes$

Ahora bien, este esquema corresponde a la negación de un esquema de contraposición, por lo que estas tablas fácilmente se adaptarían a la búsqueda de soluciones a problemas abductivos en contextos de relevancia. 
En otro orden de cosas, ya hemos visto antes la tensión entre las opciones de completud y expresividad, por así decirlo. Aunque se aspire a la máxima expresividad y la completud sea deseable, dado que los procesos de descubrimiento se pueden considerar de carácter interactivo, difícilmente serán representables mediante sistemas de lógica clásica que sean correctos y completos. A este respecto, Goldin y Wegner (2002) proponen sistemas de lógica paraconsistente como genuinos representantes de lo que llaman "computación interactiva", en cuanto opuesta a la "computación algorítmica" (modelada por máquinas de Turing), lo cual requiere la abducción y las lógicas no monótonas. Según se formule la noción misma de abducción, cabría esperar que desde una misma teoría se pudieran obtener inferencias que resultan incompatibles; por ello, concluyen estos autores, la paraconsistencia viene a ser una propiedad necesaria para una lógica que pretenda modelar la computación interactiva. Ahora bien, se han establecido tablas semánticas específicas para lógica paraconsistente, por ejemplo, en Carnielli 2006, donde se definen reglas de tablas para los sistemas LFI (logics of formal inconsistence). El lenguaje correspondiente contiene una conectiva de inconsistencia $\bullet$ (entendiendo que $\bullet A$ se interpreta como " $A$ es incierta", " $A$ es no conclusiva", etc.) y algunas reglas para la formación de las tablas se ocupan de su tratamiento. La ventaja del uso de estas tablas para el estudio de la abducción consiste en la posibilidad de búsqueda de explicaciones en contextos donde la paraconsistencia está presente, lo que no es factible en el modelo de Aliseda. ${ }^{9}$

Así, entonces, la utilización del método de las tablas semánticas para la búsqueda de soluciones a problemas abductivos constituye una buena propuesta; las tablas semánticas nos vienen a decir cómo proceder a la búsqueda sistemática de soluciones, cómo ofrecer todas las soluciones posibles, incluso cómo desechar algunas indeseables. Los buenos resultados en el nivel de lógica clásica de este procedimiento para obtener inferencias "hacia atrás" no deberían impedir que se usen en relación con la abducción en contextos inferenciales regidos por otras lógicas. Los ejemplos manejados para lógicas modales y lógicas no clásicas constituyen una muestra de la potencialidad de estos métodos.

${ }^{9}$ En Carnielli 2006 aparecen comentarios que se pueden entender como críticos al modelo presentado en Aliseda 1997. No obstante, "la abducción explicativa consistente", a la que se presta buena atención en Aliseda 2006, por sus mismas características, no pretende dar explicaciones en contextos que podríamos llamar "paraconsistentes". 


\section{BIBLIOGRAFÍA}

Aliseda, A., 2006, Abductive Reasoning: Logical Investigations into Discovery and Explanation, Springer, Dordrecht (Synthese Library, 330).

- 1997, Seeking Explanations: Abduction in Logic, Philosophy of Science and Artificial Intelligence, Universidad de Amsterdam, Amsterdam (ILLc Dissertation Series 1997-4).

Carnielli, W., 2006, "Surviving Abduction", Logic Journal of the IGPL, vol. 14, no. 2, pp. 237-256.

D'Agostino, M., M. Finger y D. Gabbay, 2008, "Cut-Based Abduction", Logic Journal of the IGPL, vol. 16, no. 6, pp. 537-560.

D'Agostino, M., D. Gabbay y K. Broda, 1999, "Tableau Method for Substructural Logics", en M. D'Agostino, D. Gabbay, R. Hähnle y J. Possega (comps.), Handbook of Tableau Methods, Kluwer, Dordrecht/Boston/Londres, pp. 397-467.

Ebbinghaus, H.D. y J. Flum, 1999, Finite Model Theory, 2a. ed., SpringerVerlag, Berlín.

Flach, P.A., 1995, Conjectures: An Inquiry Concerning the Logic of Induction, tesis doctoral, Tilburg University, Tilburg.

Gochet, P. y P. Gribomont, 2006, "Epistemic Logic", en D. Gabbay y J. Woods (comps.), Handbook of the History of Logic, Elsevier, Amsterdam, vol. 7, pp. 99-195.

Goldin, D. y P. Wegner, 2002, "Paraconsistency of Interactive Computation", en Workshop on Paraconsistency Computational Logic, <http: //www.cs.brown.edu/ pw/> [consultado: 29/06/2009].

Hintikka, J., 1999, "What Is Abduction? The Fundamental Problem of Contemporary Epistemology", Inquiry as Inquiry: A Logic of Scientific Discovery, Kluwer, Dordrecht (Jaakko Hintikka Selected Papers, 5), pp. 91-113.

Keiff, L., 2007, Le Pluralisme dialogique, tesis doctoral en filosofía, Université Charles de Gaulle Lille3, Lille.

Makinson, D., 2005, Bridges from Classical to Nonmonotonic Logic, King's College, Londres (Texts in Computing Series, 5).

Nepomuceno, A. y F. Soler, 2009, “Defining Inferential Contexts: Deduction and Abduction", en W. Carnielli, M. Coniglio e I. D'Ottaviano (comps.), The Many Sides of Logic, College Publications, Londres, pp. 511-529.

- 2007, "Metamodeling Abduction", Theoria, vol. 22-23, no. 60, pp. 285-293.

Palau, G., 2002, Introducción filosófica a las lógicas no clásicas, Universidad de Buenos Aires/Gedisa, Barcelona.

Recibido el 19 de enero de 2009; revisado el 3 julio de 2009; aceptado el 19 de agosto de 2009. 